\title{
Analysis and Simulations of LAMBDA Integer Ambiguity Resolution Algorithm
}

\author{
Shengge Zhang, Naiping Cheng and Shuyan Ni \\ Department of electronic and optical engineering of the University of Space Engineering, No.1 Bayi Road, Huairou District, \\ Beijing 101400, China
}

\begin{abstract}
In the data processing with carrier phase, how to obtain the ambiguity value of the carrier phase accurately and efficiently is an important problem in the precision positioning data processing. This paper summarizes the processing of the integer ambiguity data.. In this paper, integer least squares algorithm and LAMBDA algorithm for integer ambiguity processing are outlined.the result of calculation result in the matlab is performed.
\end{abstract}

Keywords-integer ambiguity resolution; matlab; integer least squares algorithm; LAMBDA

\section{INTRODUCTION}

In 1993, Professor Teunissen[1] of Delft University in Holland proposed the least square integer ambiguity correlation adjustment(Leastsquare AMBiguity Decorrelation Adjustment), referred to as LAMBDA. This method can narrow the scope of search and speed up the search process. It is the most successful method of ambiguity search in fast static positioning, and has been widely adopted.The LAMBDA method is a way to solve the nteger Ambiguity Resolution base on the least square method.it was widely used in Carrier phase observation.

\section{LEAST SQUARE ESTIMATION}

The carrier phase observation[2] model is shown in formula in which $y$ is $n \times 1$ dimension carrier phase measurement, $a$ is $m \times 1$ dimension integer ambiguity, $b$ is $t \times 1$ baseline vector, $A, B$ are the corresponding integer ambiguity degree and baseline vector coefficient matrix..

$$
y=A a+B b+v
$$

The least squares algorithm[3] obtains the estimated value of the parameters by minimizing the sum of squares of the errors. The least squares algorithm is used to estimate the unknown quantity of the carrier phase observation model. Then the standard least squares estimate is converted to integer least squares estimation. Integer least squares model is shown in formula.

where $Q_{y}$ is the self covariance matrix[4] of the carrier phase observations, and $a$ must be integers.

$$
\min _{a, b}\|y-A \boldsymbol{a}-B \boldsymbol{b}\|_{Q_{y}}^{2}
$$

Orthonormal decomposition (2) gives:

$$
\begin{aligned}
& \min _{a, b}\|y-A \boldsymbol{a}-B \boldsymbol{b}\|_{Q_{y}}^{2} \\
& =\|\bar{e}\|_{Q_{y}^{-1}}^{2}+\|\hat{b}-b\|_{Q_{Q^{-1}}^{2}}^{2}+\|\hat{a}-a\|_{Q_{a}^{-1}}^{2} \\
& \hat{a} \in Z^{n}, \hat{b} \in R^{t}
\end{aligned}
$$

$\hat{e}$ is the residuals of the general least squares, and $\hat{e}=y-\hat{A \boldsymbol{a}}-\hat{B \boldsymbol{b}}$, Its quadratic form is fixed value.so the Equation is maximized when $\|\hat{a}-a\|_{Q_{a}^{-1}}^{2}$ and $b=\hat{b}$.

$$
\begin{aligned}
& \min _{a, b}\|y-A \boldsymbol{a}-B \boldsymbol{b}\|_{Q_{y}}^{2} \\
& =\|\hat{e}\|_{Q_{y}^{-1}}^{2}+\|\hat{a}-\hat{a}\|_{Q_{a}^{-1}}^{2}
\end{aligned}
$$

The algorithm of integer least squares estimation is usually divided into two steps[5]:

(1)In the first step, the integer property of the ambiguities a is disregarded and the so-called float LS estimates together with their variance-covariance matrix are computed.

$$
\left[\begin{array}{c}
\hat{a} \\
\hat{b}
\end{array}\right]\left[\begin{array}{cc}
Q_{\hat{a}} & Q_{\hat{a} \hat{b}} \\
Q_{\hat{b} \hat{a}} & Q_{\hat{b} \hat{b}}
\end{array}\right] \hat{a} \in R^{n}, \hat{b} \in R^{t}
$$

(2) In the second step, the float ambiguity estimate $\hat{a}$ is used to compute the corresponding integerambiguity estimate, denoted as:

$$
\min (\hat{a}-a)^{T} Q_{\hat{a}}^{-1}(\hat{a}-a)
$$


(3) The third step is optional. It consists of deciding whether or not to accept the integer solution once integer estimates of the ambiguities have been computed. Several such tests have been proposed in the literature, cf. (Abidin 1993; Chen 1997; Euler and Schaffrin 1991; Han and Rizos 1996; Han 1997; Landau and Euler 1992; Tiberius and De Jonge 1995; Wang et al. 1998). Examples currently used in practice include the Ratio Test, the F-Ratio Test, the Difference Test and the Projector Test. These and other tests can be cast in the framework of Integer Aperture estimation, (Verhagen and Teunissen 2006; Teunissen and Verhagen 2011), which unifies steps 2 and 3 as described here.

\section{LAMBDA INTEGER AMBIGUITY FIXING ALgORITHM}

\section{A. Basic Principle of Algorithm}

In the process of integer ambiguity resolution by using the least square method, the search ellipse caused by the high correlation between each degree of ambiguity is too narrow, which is not conducive to the calculation of the ambiguity of the whole week. In order to improve the search efficiency, the integer ambiguity is transformed accordingly to reduce the correlation, so that the search ellipse is closer to the sphere. The basic principles are as follows:

Find a matrix $Z$ to satisfy: (1).All elements in A are integers; (2) $\operatorname{det}(Z)= \pm 1$;(3)invertible integer transform, that is, the integer properties of the parameters before and after transformation remain unchanged. The covariance matrix of the transformed parameters is close to the diagonal matrix as far as possible.

(1)the integer ambiguity is transformed as follows:

$$
Z=Z a, \hat{z}=Z \hat{a}, Q_{\hat{z}}=Z Q_{\hat{a}} Z^{T}
$$

(2)search the A that minimizes the target function:

$$
\min (\hat{z}-z)^{T} Q_{\hat{z}}^{-1}(\hat{z}-z)
$$

(3)The inverse transform yields a fixed solution of the original ambiguity.:

At present, the $\mathrm{Z}$ transform used in LAMBDA algorithm mainly includes: (1).iterative algorithm; (2)joint decorrelation algorithm; (3)Gauss Z transform..

\section{B. The Principle of Integer Z Transformation}

\section{1) Iterative Algorithm .}

The iterative algorithm decomposes the positive definite covariance matrix and iterates through a series of rules. The calculation of the integer transformation matrix is completed. The basic steps are as follows:

(1)The symmetric positive definite matrix $Q_{a}$ decomposes the lower triangles into $L D L^{T}$ forms, where $\mathrm{L}$ is the unit lower triangular matrix and $D$ is the diagonal matrix.assume $Q_{0}=Q_{\hat{a}}$.

$$
\begin{gathered}
Q_{0}=L_{1} D_{1} L_{1}^{T} \\
Q_{m}=\left[L_{1}^{-1}\right] Q_{0}\left[L_{1}^{-T}\right]
\end{gathered}
$$

Where $\left[^{\circ}\right]$ means rounding all elements of the matrix rounded off.

(2) The symmetric positive definite matri $Q_{m}$ is decomposed into $U D U^{T}$ form in the upper triangulation, where $U$ is the upper triangular matrix and $D$ is diagonal matrix.

$$
\begin{gathered}
Q_{0}=U_{1} D_{1} U_{1}^{T} \\
Q_{m}=\left[U_{1}^{-1}\right] Q_{0}\left[U_{1}^{-T}\right]
\end{gathered}
$$

Repeat step $1 \& 2$ in order to To minimize the correlation between ambiguity. It stops until the $\left[L_{1}^{-1}\right]$ and $\left[U_{1}^{-1}\right]$ generated by a decomposition are the unit matrix. Assuming iterations $\mathrm{n}$ times, the final $\mathrm{Z}$ transformation matrix is

$$
Z=\left[U_{n}^{-1}\right]\left[L_{n}^{-1}\right]\left[U_{n-1}^{-1}\right]\left[L_{n-1}^{-1}\right] \mathrm{L}\left[U_{1}^{-1}\right]\left[L_{1}^{-1}\right]
$$

2) Joint De-correlation Algorithms .

The steps of this algorithms are: determining the swap matrix $L$ and the unit lower triangle matrix $H$ by $Q_{\mathbb{\$}}$, and calculating the joint decorrelation matrix $[U]$ by the matrix product. Repeat the steps until a certain $[U]$ is a unit matrix, stop it, set the iteration, and finally the transformation matrix is:

$$
Z=\left[U_{n}\right]\left[U_{n-1}\right] \mathrm{K}\left[U_{1}\right]
$$

\section{Simulation And Test}

Assuming

$$
\text { afloat }=\left[\begin{array}{c}
5.5 \\
-2.6 \\
6
\end{array}\right] \text { and } Q=\left[\begin{array}{ccc}
8.8 & 4 & -7.7 \\
4 & 2.6 & -4.9 \\
-7.7 & -4.9 & 13
\end{array}\right]
$$

Fixing the ambiguity by LAMBDA algorithms 


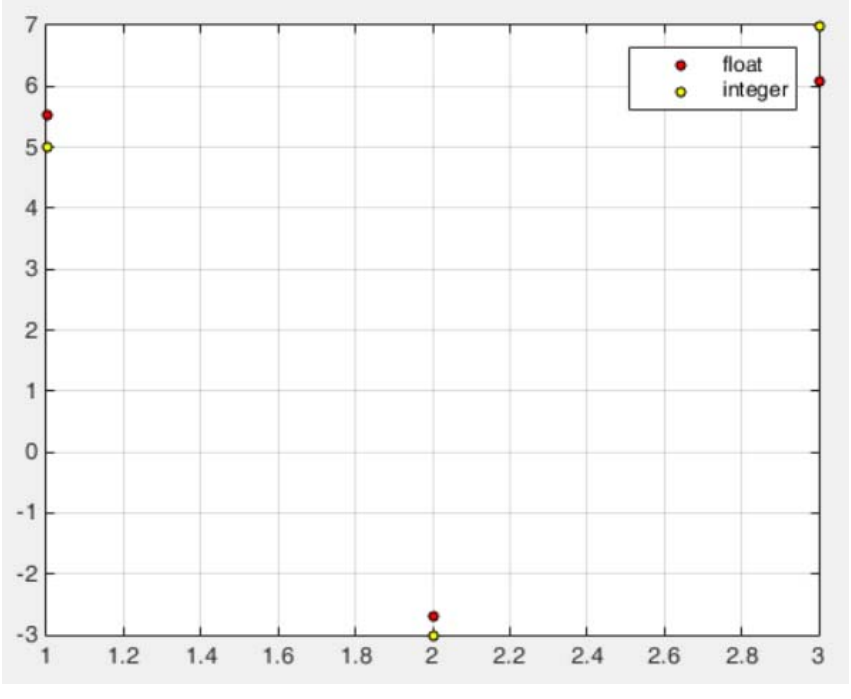

FIGURE I. THE RESULT OF LAMBDA ALGORITHM

\section{CONCLUSION}

The LAMBDA algorithm can fix the integer in a good area,it can be used in Carrier phase measurement in order to improve the accuracy of the other algorithms in GNSS system

\section{REFERENCES}

[1] Teunissen P J G. The least-squares ambiguity decorrelation adjustment: a method for fast GPS integer ambiguity estimation[J]. Journal of Geodesy, 1995, 70(1-2):65-82.

[2] Blewitt G. Carrier Phase Ambiguity Resolution for the Global Positioning System Applied to Geodetic Baselines up to $2000 \mathrm{~km}$. Journal of Geophysical Research, Vol. 94, No. B8, pp. 10.187-10.203[J]. Journal of Geophysical Research Atmospheres, 1989, 94(B8).

[3] Raquet J F. Development of a Method for Kinematic GPS Carrier-Phase Ambiguity Resolution Using Multiple Reference Receivers[J]. Dissertation Abstracts International, Volume: 59-09, Section: B, page: 5008.;Adviser: Gerard Lachape, 1998.

[4] Forssell B, Martinneira M, Harrisz R A. Carrier phase ambiguity resolution in GNSS-2[J]. Proceedings of Ion Gps, 1997:1727-1736.

[5] Teunissen P J G. The Lambda Method for the GNSS Compass[J]. Artificial Satellites, 2006, 41(3):89-103. 